\title{
Adaptação Portuguesa da Escala de Thriving na Adolescência
}

\author{
Ana Soares ${ }^{1}$, José L. Pais-Ribeiro(D) \\ Universidade do Porto, Porto, Portugal \\ Isabel Silva(D) \\ Universidade Fernando Pessoa, Porto, Portugal
}

\section{RESUMO}

No âmbito da perspectiva do Desenvolvimento Positivo dos Adolescentes, a investigação centra-se no que define não apenas o desenvolvimento normal ou adequado, mas um desenvolvimento ótimo, ou Thriving. O conceito engloba indicadores de Thriving que representam a interação relacional dinâmica e bidirecional do adolescente e contextos desenvolvimentais ao longo do tempo, estado e processo de desenvolvimento. Este estudo teve como objetivo a adaptação à população Portuguesa de Escala de Thriving na Adolescência. Foi avaliada uma amostra de conveniência constituída por 503 estudantes, com idades compreendidas entre os 13 e 19 anos $(M=15,92$; $D P=1,17)$, maioritariamente do género feminino $(63 \%)$. Originalmente desenvolvida na perspetiva do modelo formativo, a Escala de Thriving inclui sete itens. A exploração das propriedades psicométricas, com recurso a análise com rede nomológica, mostra uma escala multidimensional com propriedades métricas adequadas. Sugere-se a utilidade deste instrumento para a compreensão de um desenvolvimento bem-sucedido na adolescência.

Palavras-chave: thriving; perspetiva do desenvolvimento positivo dos adolescentes; estudo de adaptação.

\section{ABSTRACT - Portuguese Adaptation of the Thriving in Adolescence Scale}

In the field of Positive Adolescent Development research focuses on what defines not only normal or adequate development but optimal development for Thriving. The concept encompasses Thriving indicators that represent a dynamic and bi-directional interplay over time between a young person and the developmental contexts, reflecting both a point-in-time status as well as a developmental process. This study aimed to adapt the Thriving in Adolescence Scale to the Portuguese population. A convenience sample consisted of 503 students aged between 13 and 19 years $(M=15.92 ; S D=1.17)$, with $63 \%$ females. Originally developed from the perspective of the formative model, the Thriving Scale includes seven items. The exploration of psychometric properties, using nomological network analysis, showed a multidimensional scale with adequate metric properties. The use of this instrument is suggested for the understanding of successful development in adolescence.

Keywords: thriving, positive adolescent development; adaptation study.

\section{RESUMEN - Adaptación Portuguesa de la Escala de Thriving en la Adolescencia}

La investigación se desenvuelve dentro de la perspectiva del Desarrollo Positivo de los Adolescentes, englobando no solo el desarrollo normal o adecuado, sino que un desarrollo óptimo, o Thriving. El concepto incluye indicadores Thriving que representan la interacción relacional dinámica y bidireccional del adolescente y contextos de desarrollo a lo largo del tiempo, reflejando tanto un estado puntual, como un proceso de desenvolvimiento. Este estudio tuvo como objetivo la adaptación a la población Portuguesa de Escala de Thriving en la Adolescencia. Se evaluó una muestra de conveniencia constituida por 503 estudiantes, con edades comprendidas entre los 13 y 19 años $(M=15,92 ; D S=1,17)$, en su mayoría mujeres (63\%). La Escala de Thriving incluye siete ítems, siendo originalmente desarrollada en la perspectiva del modelo formativo. La exploración de las propiedades psicométricas mediante el análisis con red nomológica, muestra una escala multidimensional con propiedades métricas adecuadas. Se sugiere que este instrumento es útil para comprender el desarrollo óptimo en la adolescencia.

Palabras clave: thriving, perspectiva del desarrollo positivo de los adolescentes; estudio de adaptación.

No âmbito do estudo do desenvolvimento na adolescência, surge, no início dos anos 90, salientando-se, no início do século XXI, a Perspetiva do Desenvolvimento Positivo da Adolescência (Lerner \& Steinberg, 2009), que propõe uma nova visão e vocabulário na abordagem da adolescência (Damon, 2004). Essa perspetiva constitui uma metateoria relacional, enquadrada pelas Teorias Sistémicas do Desenvolvimento, que apresenta

${ }^{1}$ Endereço para correspondência: Avenida Joaquim Ferreira Araújo, 4730-280, Marrancos, Vila Verde, Portugal. Tel.: (351) 96305-9627. Fax: (351) 25338-2437. E-mail: anasofiabsoares@gmail.com

Artigo derivado de Tese de Doutoramento de Ana Sofia Barros Soares com orientação de José Luís Pais Ribeiro e Isabel Silva, defendida em 2019 no Programa Doutoral em Psicologia da Faculdade de Psicologia e de Ciências da Educação da Universidade do Porto. 
um quadro conceptual e metodológico com uma conceção do adolescente baseada nas suas forças, com foco nos fatores que favorecem um desenvolvimento ótimo (Benson, Scales, Hamilton, \& Sesma, 2006; Bonell et al., 2016; Damon, 2004; Lerner \& Steinberg, 2009).

Distingue-se como uma alternativa conceptual aos modelos de saúde com foco em problemas e défices do desenvolvimento que caracterizaram a investigação sobre o desenvolvimento na adolescência durante a primeira metade do século XX (Lerner \& Steinberg, 2009). A saúde do adolescente compreendida como a ausência de sintomas, patologia ou comportamentos que comprometem a saúde, torna-se uma visão incompleta (Benson, 2007). Desse modo, a investigação na adolescência tem procurado focar o que define não apenas um desenvolvimento normal ou adequado, mas um desenvolvimento ótimo, isto é, thriving (Benson, et al., 2006). Thriving poderia traduzir-se por prosperar, florescer, desenvolver, confundindo-se, se traduzido, com conceitos já existentes, pelo que se decidiu manter o termo original.

Para compreender o conceito de thriving, é necessário compreender as características que definem as Teorias Sistémicas do Desenvolvimento (Ford \& Lerner, 1992; Gottlieb, 1997; Lerner \& Steinberg, 2009; Overton, 2006). Estas são configuradas por uma metateoria relacional do desenvolvimento humano, propondo uma abordagem integrada na compreensão do desenvolvimento que envolve todos os níveis de organização desde o biológico e psicológico, ao cultural e histórico. Essa regulação compreende relações individuo $\leftrightarrow$ contexto mutuamente influentes entre todos os níveis do sistema de desenvolvimento (isto é, relações bidirecionais). Como consequência, o sistema de desenvolvimento é caracterizado por um potencial de mudança sistemática, pela plasticidade, recurso fundamental para o desenvolvimento humano. Esses fundamentos levam ao pressuposto central de que o desenvolvimento humano positivo, isto é, thriving, pode ser alcançado por meio da aplicação das ciências do desenvolvimento a ações planificadas através da articulação das características dos indivíduos e das ecologias.

Bundick, Yeager, King e Damon (2010, p.882) sugerem que thriving enquanto "a developmental goal or telos", com foco nos aspetos do desenvolvimento para além da relativa ausência de patologia, e para além da mera competência ou simples realização de tarefas desenvolvimentais (developmental tasks), consiste numa "teoria de um desenvolvimento ótimo (não apenas adequado)" (Bundick et al., 2010, p.891). Essa atenção a um desenvolvimento excelente ou ótimo vai ao encontro do trabalho do Search Institute na definição sobre o que significa thrive developmentally (Benson \& Scales, 2009; Scales, Benson, Leffert, \& Blyth, 2000; Sesma, Mannes, \& Scales, 2013). Thriving é expressão específica do desenvolvimento positivo na adolescência (Benson \& Scales, 2009, p.90).
Benson e Scales (2009) explicam que thriving representa a interação relacional dinâmica e bidirecional ao longo do tempo do adolescente e contextos desenvolvimentais; constituindo processo de experiência de um equilíbrio entre continuidade e descontinuidade do desenvolvimento que é ótimo/ideal para as relações entre um indivíduo e os seus contextos; e reflete, quer onde o adolescente está atualmente na sua jornada, quer se está no tipo de caminho para chegar ao que pode ser chamado de regulações desenvolvimentais adaptativas exemplares. Isto é, o construto refere-se tanto ao atual desenvolvimento bem-sucedido, "a localização" em indicadores particulares, como ao "caminho" de desenvolvimento da pessoa, ou seja, refere-se a uma "trajetória desenvolvimental ascendente" (Benson \& Scales, 2009, p.90). Os autores notam que essa definição de thriving reflete complexidade de equilíbrio e plasticidade entre a pessoa e contextos, continuidade e descontinuidade, estado e processo, pelo que é sugerido descrever um adolescente "at any point in time as more or less thriving oriented, rather than as thriving or not" (Benson \& Scales, 2009, p.90). Desse modo, thriving é considerado um processo de desenvolvimento, o qual pode ser caracterizado pela orientation toward life (orientação esta que reflete os princípios do processo de thriving). De salientar que, embora resultados de um estado positivo constituam importantes parâmetros, é essencial compreender quais os indicadores que, durante a adolescência, nos revelam que a pessoa está a realizar um percurso no sentido de um futuro bem-sucedido, pelo que Benson e Scales (2009, p.90) notam, Thriving refere-se ao "caminho em que se está".

Comparativamente à substancial investigação sobre comportamentos de risco e comportamentos negativos na adolescência, adequados ou competência, a pesquisa sobre o conceito de thriving na adolescência continua em estudo (consultar, por exemplo, os estudos de Benson \& Scales, 2009; Brown, Arnold, Fletcher, \& Standage, 2017; Bundick et al., 2010; Lerner, Lerner, von Eye, Bowers, \& Lewin-Bizan, 2011; Scales, Benson, \& Roehlkepartain, 2011; Wiese, Tay, Su, \& Diener, 2018), observando-se uma falta de consenso na investigação sobre o que consiste thriving, do que este inclui, e como pode ser medido. Sesma, Mannes e Scales (2013) notam que uma maior dificuldade em alcançar um consenso sobre o que constitui thriving na adolescência, comparativamente a comportamentos de risco que são necessários atender na adolescência, se deve ao facto de indicadores de thriving refletirem mais uma perspetiva moral e contextual particular - comparativamente a indicadores de risco (cujos efeitos são notórios independentemente do contexto cultural ou orientação moral, isto é, como por exemplo, o efeito prejudicial na saúde do consumo de tabaco ou substâncias ilícitas) -, sendo menos provável a universalidade do conceito.

A investigação nesse campo de estudo levou à emergência de uma diversidade de escalas de avaliação da 
experiência de thriving, dirigidas tanto a crianças e adolescentes como a adultos. De notar que, as diferentes escalas desenvolvidas refletem em si os diferentes modelos de abordagem ao conceito. Não pretendendo uma análise exaustiva das diversas escalas desenvolvidas para avaliar a experiência de thriving, salientam-se, a título de exemplo, estudos que permitiram o desenvolvimento das escalas Comprehensive Inventory of Thriving (CIT) e Brief Inventory of Thriving (BIT) (Su, Tay, \& Diener, 2014; Wiese et al., 2018), entre outros (Duan, Guan, \& Gan, 2016; Geldhof, Bowers, \& Lerner, 2013).

O conceito de thriving tem sido amplamente estudado no Search Institute com variações que refletem o desenvolvimento de medidas mais aprofundadas (Scales et al., 2011), ou medidas mais breves da experiência de thriving (Scales et al., 2000). Neste estudo, focamos o desenvolvimento de uma medida breve de avaliação da experiência de thriving proposta pelo Search Institute, cujo objetivo consiste na sua utilização enquanto medida de resultado de um desenvolvimento ótimo durante a adolescência. Essa escala está inserida e constitui parte integrante do Questionário A\&B, o qual avalia a experiência de forças, recursos e qualidades dos adolescentes, explorados tanto a nível individual como contextual, sugerindo-se que a experiência destes está relacionada com um desenvolvimento bem-sucedido (Benson, Scales, \& Syvertsen, 2011; Soares, Pais-Ribeiro, \& Silva, 2019a, 2019b). Desse modo, thriving, ou especificamente os indicadores de thriving propostos pelo Search Institute representam o estado e processo decorrente da experiência de importantes forças e recursos presentes na vida dos adolescentes que os conduzem desse modo à experiência de thriving - ou, por outras palavras, a resultados de um desenvolvimento ótimo.

Pretende-se, neste estudo, explorar a experiência de thriving, quer como um estado pontual, quer como um processo de desenvolvimento (Benson \& Scales, 2009; Benson \& Scales, 2011; Sesma, et al., 2013) utilizando-se para tal uma medida breve da sua experiência. Desse modo, o Search Institute focou sete indicadores de thriving na adolescência: Sucesso Escolar, Liderança, Ajudar Outros, Preservar Saúde, Adiar Gratificação, Valorizar Diversidade e Superar Adversidade (Scales et al., 2000). Esses indicadores foram selecionados pela sua relação com diversos resultados positivos ao nível físico, socioemocional, psicológico e cognitivo ao longo do desenvolvimento, associações estas que se verificam nos adolescentes em diversos contextos socioeconómicos, grupos étnicos e géneros. Operacionalizados como estado, sugerem processos globais do desenvolvimento. Por sua vez, refletem no seu todo que os adolescentes realizaram, pelo menos de uma forma adequada ou até mesmo excelente, uma série de tarefas do desenvolvimento (developmental tasks) concebidas como importantes para todos adolescentes. Conceptualmente não é esperado que os adolescentes apresentem elevados resultados em todos os marcadores para se considerar que estes experimentem thriving. Como observado anteriormente, a proposição de que thriving reflete a fusão da pessoa e contexto em relações mutuamente benéficas, implica que existem vários caminhos, ou combinações de marcadores, que conduzem à experiência de thriving (Scales et al., 2000; Scales \& Leffert, 2004; Sesma et al., 2013).

O presente estudo adotou um desenho observacional descritivo transversal, de carácter exploratório, com o objetivo de adaptar a Escala de Thriving na Adolescência à população portuguesa. Reproduzimos os procedimentos utilizados na validação da escala original com o propósito de manter uma equivalência conceptual e métrica que reflita os pressupostos teóricos estabelecidos pelo modelo original.

\section{Método}

\section{Participantes}

Foi avaliada uma amostra de conveniência constituída por 503 estudantes que se encontravam a frequentar o sistema de ensino regular entre o $9^{\circ}$ e o $12^{\circ}$ ano de escolaridade de uma escola pública da região Norte de Portugal, com idades compreendidas entre os 13 e 19 anos de idade $(M=15,92 ; D P=1,17)$, maioritariamente do género feminino (63\%). Como critério de inclusão, considerou-se a totalidade dos estudantes que se encontravam a frequentar o ensino regular entre o $9^{\circ}$ e o $12^{\circ}$ ano de escolaridade, considerando-se como critério de exclusão os alunos que se encontravam a frequentar o ensino profissionalizante, uma vez que contemplam padrões de avaliação distintos.

\section{Material}

\section{Escala de Thriving}

A Escala de Thriving é parte integrante do questionário Perfis da Vida de Estudante: Atitudes e Comportamentos - $\mathrm{A} \& \mathrm{~B}^{\circledR 2}$ desenvolvido pelo Search Institute - Profile of Student Life: Attitudes and Behaviors ${ }^{\circledR}$ (A\&B) (Benson, Leffert, Scales, \& Blyth, 1998; Leffert et al., 1998; Search Institute, 2019; Soares, et al., 2019a, 2019b), na perspectiva do modelo formativo (Coltman, Devinney, Midgley, \& Veniak, 2008), e dirigido a adolescentes com idades compreendidas dos 12 aos 18 anos em contexto escolar. No presente estudo, foram utilizados apenas os itens que constituem a Escala de Thriving, sendo objeto de adaptação transcultural, assim como questões demográficas. 
O Search Institute focou sete indicadores de thriving na adolescência (Scales et al., 2000). Os indicadores de thriving são medidos utilizando-se itens individuais (a avaliação inclui sete itens). A avaliação inclui os seguintes de indicadores de thriving: 1. Sucesso Escolar - Avaliado por meio de autorrelato de resultados escolares. Os adolescentes afirmam os resultados escolares numa escala Tipo Likert com nove alternativas de resposta entre "Maioritariamente muito bom (17 a 20)" a "Maioritariamente insuficiente (0 a 9)". 2. Liderança - Refere-se a designações formais ou de reconhecimento público. Os adolescentes indicam numa escala tipo Likert decinco posições, entre "1 = nunca" a " $5=$ cinco ou mais vezes", o número de vezes durante os últimos 12 meses em que foram lideres em um grupo ou organização. 3. Ajudar os Outros - Avaliado pelo número de horas em uma semana normal que os adolescentes afirmam dedicar a ajudar outras pessoas sem receber remuneração, numa escala de seis pontos tipo Likert, na qual as opções de resposta variam de "1 = nenhuma" a "6 = 11 ou mais horas por semana". 4. Preservar Saúde Física - Avaliação por meio de autorrelato relativamente a alimentação saudável e exercício físico. Consiste numa escala tipo Likert de cinco pontos aos quais os adolescentes indicam em qua medida "1 = sou muito assim" a "5 = não sou nada assim", relativamente a "cuidar bem do meu corpo (tal como comer comida saudável, praticar exercício físico regularmente e comer três boas refeições por dia)". 5. Adiar a Gratificação - Avaliação por meio de uma escala tipo Likert de cinco pontos, variando de " $1=$ sou muito assim" a "5 = não sou nada assim", relativamente à probabilidade de o adolescente poupar dinheiro para algo especial, em vez de gastar tudo imediatamente. 6. Valorizar a Diversidade - Avaliação por meio de uma escala tipo Likert de cinco pontos na qual o adolescente indica em que medida "1 = nada importante" a " $5=$ extremamente importante" "conhecer pessoas de uma raça ou grupo étnico diferente do meu". 7. Superar a Adversidade - Avaliada por meio do autorrelato do adolescente numa escala tipo Likert de cinco pontos variando de "1 = sou muito assim" a "5 = não sou nada assim", na qual o adolescente afirma em que medida as pessoas que o conhecem diriam que desiste "quando as coisas se tornam difíceis".

Existem duas abordagens para descrever a experiência de thriving (Scales et al., 2000) 1. Essa escala pode ser analisada assumindo-se que se trata de uma escala do tipo Likert, sendo que o valor obtido em cada um dos indicadores é interpretado da seguinte forma: quanto mais elevada a pontuação, maior a experiência de thriving. Essa análise inclui também a constituição de um Índice de Thriving, composto pela soma das respostas de cada item, isto é, de cada indicador individual, permitindo a análise de experiência de thriving global (do mesmo modo, elevada pontuação expressa uma experiência superior de thriving); 2. A escala permite, ainda, que, para fins de comunicação, a pontuação de cada Indicador de
Thriving seja considerada como uma variável dicotómica, isto é, o adolescente experimenta ou não experimenta o Indicador de Thriving. Da pontuação de cada um dos sete indicadores individuais de thriving resulta também a composição do Índice de Thriving, o qual descreve o número médio de Indicadores de thriving que cada adolescente experimenta.

Satisfação com a Vida - Avaliada pela Escala de Satisfação com a Vida (SWLS), desenvolvida por Diener, Emmons, Larsen e Griffin (1985) e Pavot e Diener (1993) e validada para Portugal por Neto, Barros e Barros (1990). Consiste numa escala de cinco itens que avaliam a satisfação com a vida global, que consistem em afirmações às quais o participante responde numa escala do tipo Likert, de sete posições entre " 1 = totalmente em desacordo" e "7 = totalmente de acordo", com resultados elevados designando maior satisfação com a vida. No presente estudo, os resultados da análise de consistência interna da escala apresentaram valores considerados aceitáveis (Cronbach, 1951), $\alpha=0,83$.

Perceção de Saúde - Avaliada com o item de Perceção Geral de Saúde constituinte do questionário SF-36 (primeiro item do questionário), desenvolvido por Ware, Snow, Kosinski e Gandek (1993) e validado para Portugal por Ferreira e Santana, (2003), o qual questiona: "Em geral, como dirias que a tua saúde é?”, com opções de resposta numa escala do tipo Likert com cinco posições, que varia entre " 1 = ótima" a " $5=$ fraca". Pontuações inferiores indicam melhor perceção de saúde.

Afeto Positivo e Afeto Negativo - Avaliado por meio de Positive and Negative Affect Schedule (PANAS), desenvolvida por Watson, Clark e Tellegen (1988) e validada para Portugal por Galinha e Pais-Ribeiro (2005). Essa escala consiste em 20 emoções que representam as categorias de emoções de Afeto Positivo e Afeto Negativo. Os participantes respondem à pergunta "Em que medida sentiste cada uma das emoções durante as últimas semanas", com cinco opções de resposta que variam de "1 = Nada ou muito ligeiramente" a " $5=$ Extremamente". Pontuações mais elevadas indicam maior experiência das emoções. Os resultados da análise de consistência interna da escala indicaram valores considerados aceitáveis (Cronbach, 1951): afeto positivo $\alpha=0,87$; afeto negativo $\alpha=0,86$.

Depressão - Avaliada com dois itens do questionário Perfis da Vida de Estudante: Atitudes e Comportamentos - A\&B. Os participantes respondem à pergunta "Com que frequência te sentiste triste ou deprimido durante o último mês?", com opções de resposta numa escala do tipo Likert com cinco posições, entre " $1=$ Nunca" e "5 = O tempo todo". O segundo item questiona "Alguma vez te tentaste suicidar?" com quatro opções de resposta que variam entre $1=N$ ão e $4=\operatorname{Sim}$, mais do que duas vezes. Pontuações mais elevadas indicam maior experiência de depressão. A análise de consistência interna dos itens revelou valores considerados aceitáveis (Cronbach, 1951), $\alpha=0,79$. 


\section{Procedimento}

\section{Procedimento Recolha de Dados}

A aprovação para a realização desta pesquisa foi concedida pela Direção Geral de Educação. Este estudo foi realizado de acordo com os regulamentos relativos à ética profissional, conforme declarado pela Ordem dos Psicólogos Portugueses (2016). A Comissão Nacional de Proteção de Dados confirmou o anonimato dos participantes na recolha de dados, dando o seu parecer favorável. Foram solicitadas autorizações para a recolha de dados ao Conselho Geral e Diretor do Agrupamento de Escolas e Professores. Pais/responsáveis legais de todos os alunos que participaram do estudo assinaram o Termo de Consentimento Livre e informado para a recolha de dados, uso e publicação dos resultados. A recolha de dados foi realizada anonimamente no contexto de sala de aula com instruções padronizadas. Os alunos a quem os pais/responsáveis legais deram consentimento para a participação, foram convidados a responder ao questionário e colocá-lo num envelope. Este estudo assegurou o anonimato e a confidencialidade dos dados e a sua utilização exclusiva destes para fins de investigação.

\section{Procedimento Adaptação Transcultural Escala Thriving}

O processo de adaptação transcultural a uma nova língua e cultura é semelhante ao de construção de um questionário (Pais-Ribeiro, 2013). No processo de adaptação transcultural, seguiram-se as recomendações da International Tests Comission (2005) e de Wild et al. (2005). A adaptação da Escala de Thriving para Português-europeu - parte integrante do questionário $\mathrm{A} \& \mathrm{~B}$-, foi realizada de acordo com estas recomendações que consistiam, de forma sucinta, nas seguintes fases: 1) preparação - obtenção de permissão para utilização do instrumento , garantia do envolvimento dos autores originais no processo nomeadamente esclarecimento sobre conceitos; 2) tradução do questionário, três traduções independentes; 3) reconciliação das traduções numa só tradução - coerência e harmonização com outras versões traduzidas; 4) retrotradução, desenvolvida por entidade independente; 5) revisão de retrotradução, com o objetivo de assegurar a equivalência conceptual da tradução com o envolvimento do autor na resolução de questões complexas, processo que permitiu a realização da fase 6) harmonização; 7) cognitive debriefing; 8) revisão dos resultados de cognitive debriefing e finalização 9) revisão, da tradução concluída; 10) relatório final; 11) relatório final do processo de tradução, essencial para interpretar conjuntos de dados derivados e informar futuras traduções do instrumento, no que respeita à harmonização. Esse processo foi concluído com clarificação e aprovação dos autores.

As recomendações da International Tests Comission (2005) focam particularmente a importância de alguns aspetos a considerar, nomeadamente: efeitos de diferenças culturais, diferenças linguísticas e gramaticais, adequação da linguagem para a população a que se dirige, adequação de técnicas de testagem de formato de resposta dos itens, convenções e procedimentos para a população a que se destina, pelo que se sugere manter um julgamento sistemático que suporte a evidência linguística e psicológica de modo a aprimorar a precisão do processo de adaptação, assim como apresentar evidência da equivalência entre as diversas versões linguísticas. Nesse contexto, a validade de conteúdo assume uma centralidade decisiva (Pais-Ribeiro, 2013). Sireci (1998) e Sireci e Faulkner-Bond (2014) descrevem a validade de conteúdo como o grau em o conteúdo do questionário é congruente com os propósitos da avaliação e inclui quarto elementos sobre a qualidade do instrumento: definição (1) representação (2) e relevância (3) do domínio, assim como (4) a adequação do processo de desenvolvimento do questionário. A validade é, por definição, "... the degree to which evidence and theory support the interpretation of test scores for proposed uses of tests" (American Educational Research Association [AERA] , American Psychological Association [APA], \& National Council on Measurement in Education [NCME], 2014, p.11). Salienta-se que a validade de todo e qualquer instrumento de avaliação não se expressa por um número: ela requer uma análise complexa que relacione diversos aspetos, nomeadamente, objetivos da avaliação, contexto, variáveis a avaliar, sujeito ou população avaliada e, essencialmente, os resultados, as consequências da avaliação (Pais-Ribeiro, 2013). A validade tornou-se uma abordagem integrada (Pais-Ribeiro, 2013), incluindo dados sobre conteúdo, critério, construto, fidelidade, assim como outros parâmetros associados à teoria dos testes, incluindo as suas consequências. (APA, AERA, NCME, 2014). Com a adoção da perspetiva unitária da validade, esta é considerada o elemento central e fundamental de qualquer técnica de avaliação (Elosua \& Iliescu, 2012; Kane, 2001; 2013; Pais-Ribeiro, 2013).

Como se referiu anteriormente, o desenvolvimento da Escala de Thriving adotou o modelo formativo, isto é, a variável latente é causada pelos indicadores (Avila, et al., 2015; Bagozzi, 2011; Coltman et al., 2008; Costa, 2015; Zumbo, Gelin, \& Hubley, 2002). De notar que, no modelo formativo a causalidade emerge do indicador para o construto, enquanto que, nos modelos reflexivos, a causalidade surge da variável latente, ou construto, para o indicador. Nesse sentido, é necessária a adoção de um modelo de medida apropriado para dar significado às relações que emergem no modelo estrutural (Avila, et al., 2015; Coltman et al., 2008). No presente estudo, realizou-se a inspeção dos dados com recurso ao que Cronbach e Meehl (1955) denominaram de rede nomológica, a qual relaciona quantidades ou propriedades observáveis umas com as outras, relaciona construtos observáveis com os teóricos e relaciona construtos teóricos 
uns com os outros de modo fazer observações integradas, realizar inferências, para evidenciar a existência de validade. Como salientam Avila et al. (2015), o significado do construto depende da sua relação com os seus indicadores, mas também da sua relação com outros construtos com os quais estará ligado e que será identificada com recurso à rede nomológica. Esse método (rede nomológica ou nomological network), proposto por Cronbach e Meehl (1955), constitui o método mais apropriado para a análise da validade, no sentido em que nela todos os aspetos, estatísticos e teóricos são considerados em simultâneo e em interação (Pais-Ribeiro, 2013). Desse modo, os itens qua constituem a escala foram objeto de análise para identificação das propriedades psicométricas do instrumento, de acordo com o procedimento descrito posteriormente em seção resultados.

\section{Resultados}

\section{Inspeção Psicométrica Escala de Thriving}

No presente estudo, como referido, realizou-se a inspeção dos dados com recurso ao que Cronbach e Meehl (1955) denominaram de rede nomológica. Adotaram-se as práticas dos autores originais e utilizou-se a versão com os itens resultantes da primeira fase da adaptação onde se identificaram aspetos da validade, conceito, conteúdo e construto. Posteriormente, realizou-se a identificação de propriedades psicométricas consideradas úteis para a validação nomeadamente a convergência e divergência com outras escalas. Analisou-se adicionalmente a consistência interna da escala.

\section{Análise de Convergência/Divergência}

A análise de convergência e divergência das variáveis utilizadas contribui para a validação da escala e são apresentadas na Tabela 1. A convergência e divergência constituem subcategorias da validade. A convergência observa-se quando a medida de um construto, que teoricamente deve estar relacionada com a medida de outro construto, exibe de fato uma relação substancial com ela; enquanto que, a divergência observa-se quando construtos que teoricamente não se relacionam, não mostram de facto relação entre eles. Esses valores são encontrados geralmente por meio da correlação (Campell \& Fiske, 1959). A convergência exprime-se por valores de correlação, sendo que, correlações desejáveis deveriam estar em valores médios a altos, ou seja, entre 0,30 e acima de 0,50 (Cohen, 1992) sem alcançar valores de 0,70 - que poderiam expressar redundância das variáveis. Os resultados apresentados na Tabela 1 sugerem que as variáveis com expressão positiva se correlacionam com a experiência de Thriving de modo positivo e as de expressão negativa se correlacionam com correlação negativa.

Tabela 1

Correlação entre a Escala de Thriving e Variáveis de Comparação $(n=503)$

\begin{tabular}{lccccc}
\hline & 1 & 2 & 3 & 4 & 5 \\
\hline Índice Thriving & & & & & \\
Perceção do Estado de Saúde & $0,25^{* *}$ & & & & \\
Satisfação com a Vida & $0,32^{* *}$ & $0,44^{* *}$ & & & \\
Afeto Positivo & $0,38^{* *}$ & $0,27^{* *}$ & $0,43^{* *}$ & & \\
Afeto Negativo & $-0,10^{*}$ & $-0,27^{* *}$ & $-0,38^{* *}$ & $-0,01$ & \\
Depressão & $-0,14^{* *}$ & $-0,34^{* *}$ & $-0,51^{* *}$ & $-0,29^{* *}$ & $0,49^{* *}$ \\
\hline
\end{tabular}

Nota. ${ }^{*} p<0,05 ;{ }^{* *} p<0,01$

\section{Análise de Consistência Interna}

Indicadores de thriving são medidos utilizando-se itens individuais, pelo que a avaliação da consistência interna não se aplica. Por sua vez, de notar que, thriving constitui um conceito multidimensional, motivo pelo qual não é esperado que os itens individuais que compõem o índice representem uma única dimensão denominada "thriving" (Scales et al., 2000). Isto é, é esperado que os resultados indiquem a variação individual de interesses e capacidades, ou seja, um adolescente pode, por exemplo, apresentar elevado sucesso escolar, dedicar-se a voluntariado e atender à sua saúde, mas não exercer posições de liderança formais. Desse modo, diferenças intraindividuais são esperadas na experiência de thriving durante o processo de desenvolvimento.

\section{Experiência de Thriving}

$\mathrm{Na}$ análise dos dados utilizando a pontuação dicotómica (isto é, experimenta, ou não experimenta thriving), os resultados sugerem que o resultado médio em Índice de Thriving (1-sete indicadores) é de experiência de 3,57 indicadores $(D P=1,35)$. Relativamente aos resultados em Índice de Thriving, verificou-se que: $1 \%$ dos adolescentes afirmam experimentar zero indicadores de thriving; $6 \%$ experimenta um indicador; $16 \%$ experimenta dois; $25 \%$ experimenta três; $28 \%$ experimenta quatro; $18 \%$ experimenta cinco; $7 \%$ experimenta seis; e apenas 
$1 \%$ revela experimentar a totalidade dos sete indicadores em estudo. A análise dos dados, utilizando essa pontuação binária, considerando-se cada indicador individual indica que: 7 \% afirma experimentar Sucesso Escolar; 29 \% Liderança; 72 \% Ajudar Outros; 53 \% Saúde; 63 \% Adiar Gratificação; 71 \% Valorizar Diversidade; e $65 \%$ revela experimentar Superar a Adversidade.

Analisando os dados de outra forma, são apresentadas na Tabela 2 as estatísticas descritivas para os resultados de experiência de Índice de Thriving e Indicadores Individuais de Thriving por género e amostra total. A análise dos dados, utilizando teste $t$, sugere a existência de diferenças estatisticamente significativas entre géneros: as raparigas revelam uma maior experiência de Valorizar Diversidade e Ajudar Outros, enquanto que, os rapazes afirmam uma experiência superior de Preservar Saúde, Liderança e Superar a Adversidade. A análise de variância (one-way ANOVA, Post-Hoc Bonferroni) sugere a existência de diferenças estatisticamente significativas na experiência de thriving em função do grau de escolaridade, relativamente à experiência global de Thriving/Índice Thriving, $F(3,498)=3,08, p<0,05\left(10^{\circ}>12^{\circ}\right)$, Sucesso Escolar, $F(3,496)=3,16, p<0,05\left(10^{\circ}>12^{\circ}\right)$, Liderança, $F(3,496)=2,62, p<0,05\left(10^{\circ}>12^{\circ}\right)$ e Adiar Gratificação, $F(3,498)=2,70, p<0,05\left(9^{\circ}>10^{\circ}, 11^{\circ}, 12^{\circ}\right)$. Não se observaram diferenças estatisticamente significativas relativamente aos restantes indicadores. Considerando-se a idade, os resultados sugerem a não existência de diferenças estatisticamente significativas na experiência de thriving nas diferentes idades em estudo.

Tabela 2

Estatísticas Descritivas para Índice Thriving e Indicadores Individuais de Thriving

\begin{tabular}{|c|c|c|c|c|c|c|c|c|}
\hline \multirow{3}{*}{ Thriving } & \multirow{3}{*}{ Escala } & \multirow{2}{*}{\multicolumn{2}{|c|}{$\begin{array}{l}\text { Amostra Total } \\
\qquad(\mathrm{N}=503)\end{array}$}} & \multicolumn{4}{|c|}{ Género } & \multirow{3}{*}{$t$} \\
\hline & & & & \multicolumn{2}{|c|}{$\begin{array}{c}\text { Masculino } \\
(n=186)\end{array}$} & \multicolumn{2}{|c|}{$\begin{array}{c}\text { Feminino } \\
(n=313)\end{array}$} & \\
\hline & & $\mathrm{M}$ & $\mathrm{DP}$ & $\mathrm{M}$ & $\mathrm{DP}$ & $\mathrm{M}$ & $\mathrm{DP}$ & \\
\hline Índice Thriving & $7-40$ & 24,85 & 3,68 & 25,02 & 3,73 & 24,78 & 3,65 & $-0,70$ \\
\hline \multicolumn{9}{|l|}{ Indicador de Thriving } \\
\hline Sucesso Escolar & $1-9$ & 6,11 & 1,45 & 6,06 & 1,49 & 6,16 & 1,43 & 0,73 \\
\hline Liderança & $1-5$ & 1,57 & 1.07 & 1,74 & 1,22 & 1,48 & 0,96 & $-2,59^{* *}$ \\
\hline Ajudar Outros & $1-6$ & 2,27 & 1,19 & 2,14 & 1,01 & 2,35 & 1,27 & $1,89^{*}$ \\
\hline Preservar Saúde & $1-5$ & 3,55 & 1,14 & 3,76 & 1,06 & 3,42 & 1,15 & $-3.30^{* *}$ \\
\hline Adiar Gratificação & $1-5$ & 3,77 & 1,13 & 3,76 & 1,06 & 3,78 & 1,15 & 0,23 \\
\hline Valorizar Diversidade & $1-5$ & 3,79 & 0,95 & 3,68 & 0,99 & 3,86 & 0,92 & $2,07^{*}$ \\
\hline Superar a Adversidade & $1-5$ & 3,87 & 1,07 & 4,01 & 0.99 & 3,81 & 0,99 & $-1,99^{*}$ \\
\hline
\end{tabular}

Nota. Elevados resultados indicam experiência superior de Thriving. ${ }^{*} p<0,05 ;{ }^{* *} p<0,01$

\section{Discussão}

A perspetiva do Desenvolvimento Positivo dos Adolescentes propiciou a investigação sobre o desenvolvimento na adolescência com foco sobre o que define não apenas um desenvolvimento normal ou adequado, mas um ótimo, ou thriving. Thriving é expressão específica do desenvolvimento positivo na adolescência (Benson \& Scales, 2009), consistindo numa "theory of optimal development" (Bundick et al., 2010). Os indicadores de thriving representam a interação relacional dinâmica e bidirecional ao longo do tempo, do adolescente e seus contextos desenvolvimentais, refletindo estado, e processo dirigido a um desenvolvimento otimamente bem-sucedido (Benson \& Scales, 2009).

Este estudo focou sete indicadores de thriving em adolescentes: Sucesso Escolar, Liderança, Ajudar Outros, Preservar Saúde, Adiar Gratificação, Valorizar Diversidade e Superar Adversidade. A Escala de Thriving constitui um instrumento de avaliação integrado na investigação de um desenvolvimento ótimo (Scales et al., 2000): como se referiu, o desenvolvimento da Escala de Thriving adotou a perspetiva do modelo formativo (Costa, 2015), motivo pelo qual se considerou, neste caso, a análise nomológica de Cronbach e Mehel (1955) o método mais apropriado para a análise da validade deste instrumento (Pais-Ribeiro, 2013). Os resultados do presente estudo sugerem que a versão portuguesa da Escala de Thriving possui características de validade similares às da versão original, pelo que se adotou uma perspetiva conservadora procurando-se manter a equivalência conceptual e métrica que reflita os pressupostos teóricos que fundamentam o modelo.

Os resultados da análise dos dados decorrente da composição do Índice de Thriving sugerem que os adolescentes experimentam em média menos de quatro Indicadores de Thriving em estudo, resultados estes similares aos apresentados por Scales, Benson, Leffert e Blyth 
(2000) numa amostra agregada de estudantes americanos. A maior percentagem de adolescentes experimenta $4(28 \%)$ a $3(25 \%)$ Indicadores de Thriving, e apenas $1 \%$ dos adolescentes afirma experimentar a totalidade dos Indicadores de Thriving que compreendem o índice. $\mathrm{Na}$ análise da experiência individual de Indicadores de Thriving, observam-se resultados superiores na experiência de Ajudar os Outros (72\%), Adiar a Gratificação (63\%), Valorizar a Diversidade (71\%), assim como Superar a Adversidade (65\%). Contudo, apenas 29\% dos adolescentes revela a experiência de Liderança. Relativamente ao indicador de Preservar a Saúde, e considerando-se a importância desse indicador no desenvolvimento, apenas $53 \%$ dos adolescentes afirma a sua experiência. De notar também, apenas 7\% dos adolescentes revela a experiência de Sucesso Escolar (isto é, resultados escolares maioritariamente entre 17 a 20/maioritariamente cincos), o que indica a importância de priorizar o desenvolvimento de estratégias dirigidas ao Sucesso Escolar.

Em adição, os resultados deste estudo sugerem que a experiência de thriving pode ser contextualmente influenciada pelo género e grau de escolaridade. Embora não se observem diferenças estatisticamente significativas entre género nos resultados globais de experiência de thriving, relativamente à análise individual de indicadores de thriving observa-se que as raparigas revelam uma maior experiência de Valorizar Diversidade e Ajudar Outros comparativamente aos rapazes. Os rapazes afirmam uma experiência superior de Preservar Saúde, Liderança e Superar a Adversidade. Os resultados sugerem que thriving pode ser contextualmente influenciado pelo grau de escolaridade, observando-se que os adolescentes que frequentam um grau de escolaridade superior revelam menor experiência de thriving global, assim como menor experiência de Sucesso Escolar, Liderança e Adiar Gratificação. Contudo, de notar que, embora se observe a existência de diferenças na experiência de thriving em função do grau de escolaridade, não se verifica uma correlação estatisticamente significativa entre a experiência de thriving e idade dos adolescentes, resultados estes que sugerem a que a experiência de thriving pode ter uma influência contextual, mas a sua experiência intrínseca ao indivíduo sugere a adequação do modelo à idade a que este se dirige.

Salientamos que, thriving constitui um conceito multidimensional, por este motivo, diferenças intraindividuais são esperadas na experiência de thriving durante o processo de desenvolvimento. Esses resultados indicam a variação individual de interesses e capacidades, isto é, um adolescente pode, por exemplo, apresentar elevado sucesso escolar, dedicar-se a voluntariado e atender à sua saúde, mas não exercer posições de liderança formais. Desse modo, a utilização de indicadores sugere que thriving pode ser alcançado por meio de diversos caminhos, sendo razoável um adolescente expressar a experiência de um maior número de alguns indicadores em detrimento de outros. A composição de um índice pretende assim refletir a variedade de experiência de indicadores que podem descrever thriving e o nível geral de thriving de um adolescente num determinado momento, representando o funcionamento de um indivíduo em vários indicadores. Desse modo, não é esperado que todos os adolescentes apresentem resultados elevados em todos os indicadores, mas que os adolescentes que revelem resultados gerais superiores no Índice de Thriving apresentem a experiência de um conjunto de indicadores que refletem a modal response de thriving, comparativamente a adolescentes com resultados gerais inferiores (Scales et al., 2000). Nem todos os contextos oferecem oportunidades para, ou esperam, a experiência de todos os indicadores de thriving pelos adolescentes em todos os momentos (Theokas et al., 2005). O resultado de thriving composto de um indivíduo reflete o seu funcionamento geral, assim como o desenvolvimento de um subconjunto de experiências socialmente valorizadas. Assim, é esperado que os resultados mostrem a variação individual em interesses e capacidades. Como referimos, Benson e Scales (2009, p.90) notam que thriving reflete complexidade de equilíbrio e plasticidade entre o adolescente e seus contextos, continuidade e descontinuidade, estado e processo, pelo que é sugerido descrever um adolescente "at any point in time as more or less thriving oriented, rather than as thriving or not" (Benson \& Scales, 2009).

Por sua vez, esses resultados de thriving permitem a identificação de indicadores relevantes para todos os adolescentes, ao mesmo tempo em que destaca a importância de indicadores específicos em adolescentes com diferentes características e em diferentes contextos. Esta variedade de relações sugere algo de distintivo na forma como alguns adolescentes experimentam ou expressam thriving, salientando-se a importância de considerar diferenças na experiência de thriving ao procurar promover um desenvolvimento positivo nos adolescentes.

Os resultados deste estudo devem ser interpretados tendo em consideração as limitações seguidamente expostas. Primeiramente, salienta-se a utilização de uma amostra de conveniência, sendo não representativa da população geral, motivo pelo qual os resultados deste estudo não podem ser generalizados à população geral. As medidas utilizadas basearam-se em autorrelato, como tal, refletem a avaliação individual. Salienta-se a importância de inclusão em estudos futuros de triangulação da medição de thriving por meio de outras fontes de informação, como pais ou professores.

Em conclusão, sugere-se com a realização deste estudo a importância do desenvolvimento de investigação dirigida a conceptualizar e medir resultados de um desenvolvimento ótimo durante a adolescência, isto é, thriving. Este estudo salienta a importância dos pressupostos definidos pela Perspetiva do Desenvolvimento Positiva dos Adolescentes, com base nas teorias sistémicas do desenvolvimento, centrando-se no que define não apenas o 
desenvolvimento normal ou adequado, mas o desenvolvimento ótimo, ou thriving. Esta atenção em determinar um resultado, ou um funcionamento excelente ou ótimo, "a developmental goal or telos" (Bundick et al., 2010, p.882) sugere a importância da utilização de indicadores de thriving que representem a interação relacional dinâmica e bidirecional do adolescente e seus contextos desenvolvimentais, que expressem tanto um estado pontual, assim como um processo de desenvolvimento dirigido ao que Benson e Scales (2009, p.90) designam de "regulações desenvolvimentais adaptativas exemplares". A utilização de indicadores de thriving, fundamentados pelo pressuposto de potencial plasticidade e diversidade e conceptualizados de um ponto de vista positivo, leva ao objetivo central que sugere a adoção da expetativa de que podem ser promovidas mudanças positivas mediante ajustamentos saudáveis entre os indivíduos e os contextos.

Conclui-se pela utilidade teórica e prática a importância da utilização de indicadores de thriving para a compreensão de um desenvolvimento otimamente bem-sucedido na adolescência.

\section{Agradecimentos}

Não há menções.

\section{Financiamento}

A presente pesquisa não recebeu nenhuma fonte de financiamento sendo custeada com recursos dos próprios autores.

\section{Contribuições dos autores}

Todos os autores contribuíram substancialmente para a elaboração do delineamento da pesquisa, análise e interpretação dos dados, bem como, para a revisão textual e aprovação da versão final deste estudo. Especificamente, Ana Sofia Barros Soares, José Luís Pais- Ribeiro e Isabel Silva participaram da redação inicial do estudo - conceitualização, investigação, visualização, análise dos dados - e redação final do trabalho - revisão e edição. Todos os autores assumem responsabilidade pública pelo conteúdo do manuscrito.

\section{Disponibilidade dos dados e materiais}

Todos os dados e sintaxes gerados e analisados durante esta pesquisa serão tratados com total sigilo devido às exigências do Comitê de Ética em Pesquisa em Seres Humanos. Porém, o conjunto de dados e sintaxes que apoiam as conclusões deste artigo estão disponíveis mediante razoável solicitação ao autor principal do estudo.

\section{Conflito de interesses}

Os autores declaram que não há conflitos de interesses.

\section{Referências}

American Educational Research Association, American Psychological Association, National Council on Measurement in Education, Joint Committee on Standards for Educational, \& Psychological Testing (US). (2014). Standards for educational and psychological testing. Washington, DC: American Educational Research Association.

Avila, M. L., Stinson, J., Kiss, A., Brandão, L. R., Uleryk, E., \& Feldman, B. M. (2015). A critical review of scoring options for clinical measurement tools. BMC Research Notes, 8(1), 612. doi: 10.1186/s13104-015-1561-6.

Bagozzi, R. (2011). Measurement and meaning in information systems and organizational research: methodological and philosophical foundations. MIS Quarterly, 35, 261-292.

Benson, P. L. (2007). Developmental assets: An overview of theory, research, and practice. Em R. K. Silbereisen \& R. M. Lerner (Eds.), Approaches to Positive Youth Development (cap. 2, pp. 33-58). Thousand Oaks, CA: Sage Publications Ltd. doi: 10.4135/9781446213803.n2

Benson, P. L., Leffert, N., Scales, P. C., \& Blyth, D. A. (1998). Beyond the "village" rhetoric: Creating healthy communities for children and adolescents. Applied Developmental Science, 2(3), 138-59. doi:10.1207/s1532480xads0203_3

Benson, P. L., \& Scales, P. C. (2009). The definition and preliminary measurement of thriving in adolescence. The Journal of Positive Psychology, 4(1), 85-104. doi: 0.1080/17439760802399240

Benson, P. L., Scales, P. C., Hamilton, S. F., \& Sesma, Jr., A. (2006). Positive youth development: Theory, research and applications. Em W. Damon, \& R. M. Lerner (Eds.), Handbook of child psychology. Theoretical models of human development (6ht Ed, vol.1, pp. 894-941). New York, NY: John Wiley. doi: 10.1002/9780470147658.chpsy0116

Benson, P. L., Scales, P. C., \& Syvertsen, A. K. (2011). The contribution of the developmental assets framework to positive youth development theory and practice. Em R. M. Lerner, J. V. Lerner, and J. B. Benson (Eds.), Advances in Child Development and Behavior (Vol. 41, pp. 197-230). Amsterdam, NL: Elsevier.

Bonell, C., Hinds, K., Dickson, K., Thomas, J., Fletcher, A., Murphy, S., ... Campbell, R. (2016). What is positive youth development and how might it reduce substance use and violence? A systematic review and synthesis of theoretical literature. BMC Public Health, 16, 135. doi:10.1186/s12889-016-2817-3

Brown, D. J., Arnold, R., Fletcher, D., \& Standage, M. (2017). Human thriving. European Psychologist., 22(3), 167-179. doi: 10.1027/10169040/a000294

Bundick, M. J., Yeager, D. S., King, P. E., \& Damon, W. (2010). Thriving across the life span. Em W. F. Overton (Ed.), Handbook of Life Span Development: Methods, Biology, Neuroscience, \& Cognitive Development (3rd ed., Vol. 1). Hoboken, NJ: John Wiley \& Sons. 
Campbell, D. T., \& Fiske, D. W. (1959). Convergent and discriminant validation by the multitrait-multimethod matrix. Psychological Bulletin, 56(2), 81-105. doi:org/10.1037/h0046016

Cohen J. (1992). A Power Primer. Psychological Bulletin, 112(1), 155-159. doi: 10.1037/0033-2909.112.1.155

Coltman, T., Devinney, T. M., Midgley, D. F., \& Veniak, S. (2008). Formative versus reflective measurement models: Two applications of formative measurement. Journal of Business Research, 61(12), 1250-1262. doi: 10.1016/j.jbusres.2008.01.013

Costa, D. S. (2015). Reflective, causal, and composite indicators of quality of life: A conceptual or an empirical distinction? Quality of Life Research, 24(9), 2057-2065. doi: 10.1007/s11136-015-0954-2

Cronbach, L. J. (1951). Coefficient alpha and the internal structure of tests. Psychometrika, 16(3), 297-334. doi: 10.1007/BF02310555

Cronbach, L. J., \& Meehl, P. E. (1955). Construct validity in psychological tests. Psychological Bulletin, 52(4), 281-302. doi: 10.1037/h0040957

Damon, W. (2004). What is positive youth development? The ANNALS of the American Academy of Political and Social Science, 591(1), 13-24. doi: $10.1177 / 0002716203260092$

Diener, E. D., Emmons, R. A., Larsen, R. J., \& Griffin, S. (1985). The satisfaction with life scale. Journal of personality assessment, 49(1), 71-75.

Duan, W., Guan, Y., \& Gan, F. (2016). Brief inventory of thriving: A comprehensive measurement of wellbeing. Chinese Sociological Dialogue, 1(1), 15-31. doi: 10.1177/2397200916665230

Elosua, P., \& Iliescu, D. (2012). Tests in Europe: Where we are and where we should go. International Journal of Testing, 12(2), 157-175. doi: org/10.1080/15305058.2012.657316

Ferreira, P., \& Santana, P. (2003). Percepção do estado de saúde e de qualidade de vida da população activa: Contributo para a definição de normas portuguesas. Revista Portuguesa de Saúde Pública, 21(2), 15-30.

Ford, D. H., \& Lerner, R. M. (1992). Developmental systems theory: An integrative approach. Newbury Park, CA: Sage Publications.

Galinha, I. C., \& Pais-Ribeiro, J. L. (2005). Contribuição para o estudo da versão portuguesa da Positive and NegativeAffect Schedule (PANAS): II-Estudo psicométrico. Análise psicológica, 23(2), 219-227.

Geldhof, G. J., Bowers, E. P., \& Lerner, R. M. (2013). Special section introduction: Thriving in context: Findings from the 4-H study of positive youth development. Journal of Youth Adolescence, 42, 1. doi:10.1007/s10964-012-9855-7

Gottlieb, G. (1997). Synthesizing nature-nurture: Prenatal roots of instinctive behavior. Mahwah, NJ: Lawrence Erlbaum

International Test Commission (2005). International guidelines on test adaptation. Recuperado de www.intestcom.org

Kane, M. (2001). Current concerns in validity theory. Journal of Educational Measurement, 38(4), 319-334. doi: 10.1111/j.1745-3984.2001. tb01130.x

Kane, M. (2013). Validating the interpretations and uses of test scores. Journal of Educational Measurement, 50(1), 1-73. doi: 10.1111/jedm.12000.

Leffert, N., Benson, P., Scales, P., Sharma, A., Drake, D., \& Blyth, D. (1998). Developmental assets: Measurement and prediction of risk behaviors among adolescents. Applied Developmental Science, 2(4), 209-230. doi: 10.1207/s1532480xads0204_4

Lerner, R. M., \& Steinberg, L. (2009). The scientific study of adolescent development. Em R. M.., Lerner \& L. Steinberg (Eds.), Handbook of adolescent psychology (3th Ed., vol1, pp. 3-14). New Jersey, US: John Wiley \& Sons, Inc. doi: 10.1002/9780470479193.adlpsy001002

Lerner, R. M., Lerner, J. V., von Eye, A., Bowers, E. P., \& Lewin-Bizan, S. (2011). Individual and contextual bases of thriving in adolescence: A view of the issues. Journal of adolescence, 34(6), 1107-1114. doi: org/10.1016/j.adolescence.2011.08.001

Ordem dos Psicólogos Portugueses (2016). Código Deontológico da Ordem dos Psicólogos Portugueses. Recuperado de https://www. ordemdospsicologos.pt/ficheiros/documentos/web_cod_deontologico_pt_revisao_2016.pdf

Overton, W. F. (2006). Developmental psychology: Philosophy, concepts, and methodology. Em W. Damon \& R. M. Lerner (Eds.), Theoretical Models of Human Development. Handbook of child psychology (5th Ed., vol.1, pp.107-188), Hoboken, NJ: Wiley.

Pais-Ribeiro, J. (2013). Medida na avaliação psicológica. Psicologia, Saúde \& Doenças, 14(1), 245-263

Pavot, W., \& Diener, E. (1993). The affective and cognitive context of self-reported measures of subjective well-being. Social Indicators Research, 28(1), 1-20.

Scales, P. C., Benson, P. L., \& Roehlkepartain, E. C. (2011). Adolescent thriving: The role of sparks, relationships, and empowerment. Journal of youth and adolescence, 40, 263-277. doi: 10.1007/s10964-010-9578-6

Scales, P. C., Benson, P. L., Leffert, N., \& Blyth, D. A. (2000). Contribution of developmental assets to the prediction of thriving among adolescents. Applied Developmental Science, 4(1), 27-46. doi: 10.1207/S1532480XADS0401_3

Scales, P. C., \& Leffert, N. (2004). Developmental assets: A synthesis of the scientific research on adolescent development (2nd Ed.). Minneapolis, MN: Search Institute.

Search Institute (2019). User guide for the Attitudes \& Behaviors survey. Recuperado de http://www.search-institute.org/sites/default/files/a/A\&BSurvey-User-Guide.pdf

Sesma Jr, A., Mannes, M., \& Scales, P. C. (2013). Positive adaptation, resilience and the developmental assets framework. Em S. Goldstein \& R. B. Brooks (Eds.) Handbook of resilience in children (cap. 25, pp. 427-442). New York, US: Springer. doi 10.1007/978-1-4614-3661$4 \_25$

Sireci, S. G. (1998). The construct of content validity. Social indicators research, 45(1-3), 83-117.

Sireci, S., \& Faulkner-Bond, M. (2014). Evidencia de validez basada en el contenido del test. Psicothema, 26(1), 100-108.

Soares, A. S. B., Pais-Ribeiro, J. L., \& Silva, I. M. S. L. (2019a). Developmental assets predictors of life satisfaction in adolescents. Frontiers in psychology, 10, 236. doi:10.3389/fpsyg.2019.00236

Soares, A. S., Pais-Ribeiro, J. L., \& Silva, I. (2019b). Adaptação portuguesa do questionário Profile of Student Life: Attitudes and Behaviors (A\&B). Submitted.

Su, R., Tay, L., \& Diener, E. (2014). The development and validation of the Comprehensive Inventory of Thriving (CIT) and the Brief Inventory of Thriving (BIT). Applied Psychology: Health and Well-Being, 6, 251-279. doi: 10.1111/aphw.12027

Theokas, C., Almerigi, J. B., Lerner, R. M., Dowling, E. M., Benson, P. L., Scales, P. C., \& von Eye, A. (2005). Conceptualizing and modeling individual and ecological asset components of thriving in early adolescence. The Journal of Early Adolescence, 25(1), 113-143. doi: $10.1177 / 0272431604272460$

Ware, J. E., Snow, K. K., Kosinski, M., \& Gandek, B. (1993). SF-36 Health Survery. Manual and interpretation guide. The Health Institute, New England Medical Center. Boston, Mass, 136.

Watson, D., Clark, L. A., \& Tellegen, A. (1988). Development and validation of brief measures of positive and negative affect: the PANAS scales. Journal of personality and social psychology, 54(6), 1063. 
Wild, D., Grove, A., Martin. M., Eremenco, S., McElroy, S., Verjee-Lorenz, A., \& Erikson, P. (2005). Principles of good practice for the translation and cultural adaptation process for patient-reported outcomes (PRO) measures: Report of the ISPOR task force for translating adaptation. Value Health, 8(2), 94-104. doi: 1098-3015/05/94 94-104

Wiese, C. W., Tay, L., Su, R., \& Diener, E. (2018). Measuring thriving across nations: Examining the measurement equivalence of the Comprehensive Inventory of Thriving (CIT) and the Brief Inventory of Thriving (BIT). Applied Psychology: Health and Well-Being, 10(1), 127-148. doi:10.1111/aphw.12119

Zumbo, B. D., Gelin, M. N., \& Hubley, A. M. (2002). The Construction and Use of Psychological Tests and Measures. Em The Psychology theme of the Encyclopedia of Life Support Systems (EOLSS) (pp.1-28). Oxford, UK: Eolss Publisher.

\section{Sobre os autores}

Ana Sofia Barros Soares é doutorada em Psicologia pela Faculdade de Psicologia e de Ciências da Educação da Universidade do Porto. Professora no ISAVE - Instituto Superior de Saúde.

José Luís Pais Ribeiro é doutorado em Psicologia pela Faculdade de Psicologia e de Ciências da Educação da Universidade do Porto. Professor Faculdade de Psicologia e de Ciências da Educação da Universidade do Porto e ISPA - Instituto Universitário. Investigador no William James Center for Research.

Isabel Silva é doutorada em Psicologia pela Faculdade de Psicologia e de Ciências da Educação da Universidade do Porto. Professora Associada na Universidade Fernando Pessoa, Porto. Coordenadora do Centro de Investigação FP-B2S e Coordenadora do Psychometry Lab.

\section{Como citar este artigo}

Soares, A., Pais-Ribeiro, J. L., \& Silva, I. (2020). Adaptação Portuguesa da Escala de Thriving na Adolescência. Avaliação Psicológica, 19(4), 371-381. http://dx.doi.org/10.15689/ap.2020.1904.17222.03 